Sciences

Vol. 06, No. 01, pp. 75-89, March 2013

\title{
FAST CONVOLUTION AND CORRELATION ALGORITHM FOR MULTIDIMENSIONAL SIGNALS
}

\author{
Emad Hmood Salman \\ Communication Eng. Dept., College of Engineering, University of Diyala \\ Email: ehs baghdad@yahoo.com \\ (Received:29/1/2012 ; Accepted:11/6/2012)
}

\begin{abstract}
The technical world is changing very rapidly. In recent three decades, the power of personal computers has increased by a factor of nearly one-thousand. By all accounts, it will increase by another factor of one-thousand in the next decade. This tremendous power has changed the way science and engineering is done, and there is no better example of this than digital signal processing (DSP).

This proposal is a fast algorithm to compute some important mathematical operations in the DSP, which are the convolution and the correlation (both linear and circular). These operations are entered in most application daily computers, cellular phones, aerospace, medical devices, mechanical statuses, images processing, TV conferences, and other. Therefore, that to get at time realization system (i.e. to reduce the delay of time in any system).

Keywords:- DSP, Fast Algorithm, Linear Convolution, Circular Convolution, Linear Correlation, Circular Correlation, Multi-Dimensional Convolution, and MultiDimensional Correlation.
\end{abstract}

\section{INTRODUCTION}

The digital signal processing is one of the most powerful technologies that shape science and engineering in the last decades. Revolutionary changes have already been made in a broad range of fields: communications, medical imaging, radar and sonar, high-fidelity music reproduction, and oil prospecting, to name just a few, each of these areas has developed a deep DSP technology, with its own algorithms, mathematics, and specialized techniques ${ }^{[1-3]}$. One of most interesting functions used of DSP is the discrete convolution, which is a valuable tool for the analysis and design of communications systems and many other engineering and scientific activities, and the other function that is not less from the convolution, which is the 
correlation, is interesting function that is very useful in noise-free (and noise-contaminated) channels ${ }^{[4-7]}$.

Some applications of typical digital signal processing in real world digital crossover audio system, interference cancellation in electrocardiography, speech coding and compression, compact-disc recording system and digital photo image enhancement. Each of previous applications and other are required rapidity and accuracy in calculations especially in the convolution and correlation calculations, the present work clears that, for any number from the dimensions, 1-D, 2-D, 3-D, ...etc.

\section{THE FAST ALGORITHM}

The fundamentals of convolution and correlation computing are same, but way of applied these fundamentals are different comparing with the traditional ways. The following sections clear that.

\subsection{Fast Convolution Algorithm}

The process of convolution is according to the following algorithm:

Step 1. Add zeros to the sequences according to the length of the resultant sequence (linear or circular).

Step 2. Indicate the start-sample in both sequences.

Step 3. Obtain the reversed sequence, then product the first sequence with the second sequence to produce the first sample of the resultant sequence.

Step 4. According to start-sample in the reversed sequence, shift this sequence in each location of other samples. Continue the process of product the first sequence with the second sequence in each case.

Step 5. The location of each resulted sample is the same as location of start-sample of the reversed sequence (note that, the start-sample of the resultant sequence produces from case of product both start-samples of the sequences).

\subsection{Fast Correlation Algorithm}

The process of correlation is according to the following algorithm:

Step 1. Add zeros to the sequences according to the length of the resultant sequence (linear or circular).

Step 2. Indicate the start-sample in the first sequence and indicate the final-sample in the second sequence. 
Step 3. According to final-sample in the second sequence, shift this sequence in each location of other samples. Continue the process of product the first sequence with the second sequence in each case.

Step 4. The location of each resulted sample is the same as location of the final-sample of the second sequence (note that the start-sample of the resultant sequence produces from case of product the start-sample with the final-sample).

\section{DEMONSTRATED EXAMPLES ABOUT THE FAST ALGORITHM}

There are four examples clear the above algorithms according to type of function:

\section{Fast Convolution 2-D:}

Assume the two matrices below:

$$
x=\left[\begin{array}{cc}
1 & \llbracket-3 \rrbracket \\
2 & 4
\end{array}\right] \text { and } h=\left[\begin{array}{ll}
\llbracket 0 \rrbracket & 2 \\
-5 & 1
\end{array}\right]
$$

In addition, $y$ is a matrix and it is equal to linear convolution of $x$ with $h$ as follows

$$
y(n 1, n 2)=\Sigma_{k 1} \Sigma_{k 2} x(k 1, k 2) h(n 1-k 1, n 2-k 2)
$$

or $\quad y(n 1, n 2)=x(n 1, n 2) * h(n 1, n 2)$

(2)

Where the symbol ${ }^{* *}$ represents the 2-D discrete convolution ${ }^{[8]}$. It clears that number of rows and columns are equal to three, in matrix $y$. So according to the fast convolution algorithm the solution as follows:

$$
\begin{aligned}
x & =\left[\begin{array}{ccc}
1 & \llbracket-3 \rrbracket & 0 \\
2 & 4 & 0 \\
0 & 0 & 0
\end{array}\right], h=\left[\begin{array}{ccc}
0 & \llbracket 0 \rrbracket & 2 \\
0 & -5 & 1 \\
0 & 0 & 0
\end{array}\right] \text { and } h \text { reverse is, } h x=\left[\begin{array}{ccc}
0 & 0 & 0 \\
1 & -5 & 0 \\
2 & \llbracket 0 \rrbracket & 0
\end{array}\right], \text { therefore } \\
y & =\left[\begin{array}{ccc}
1 & \llbracket-3 \rrbracket & 0 \\
2 & 4 & 0 \\
0 & 0 & 0
\end{array}\right] \times\left[\begin{array}{ccc}
0 & 0 & 0 \\
1 & -5 & 0 \\
2 & \llbracket 0 \rrbracket & 0
\end{array}\right]=0+0+0+2-20+0+0+0+0=-18 \\
y & =\left[\begin{array}{ccc}
1 & \llbracket-3 \rrbracket & 0 \\
2 & 4 & 0 \\
0 & 0 & 0
\end{array}\right] \times\left[\begin{array}{ccc}
0 & 0 & 0 \\
-5 & 0 & 1 \\
\llbracket 0 \rrbracket & 0 & 2
\end{array}\right]=0+0+0-10+0+0+0+0+0=-10 \\
y & =\left[\begin{array}{ccc}
1 & \llbracket-3 \rrbracket & 0 \\
2 & 4 & 0 \\
0 & 0 & 0
\end{array}\right] \times\left[\begin{array}{ccc}
-5 & 0 & 1 \\
\mathbb{0} \rrbracket & 0 & 2 \\
0 & 0 & 0
\end{array}\right]=-5+0+0+0+0+0+0+0+0=-5 \\
y & =\left[\begin{array}{ccc}
1 & \llbracket-3 \rrbracket & 0 \\
2 & 4 & 0 \\
0 & 0 & 0
\end{array}\right] \times\left[\begin{array}{ccc}
\llbracket 0 \rrbracket & 0 & 2 \\
0 & 0 & 0 \\
-5 & 0 & 1
\end{array}\right]=0+0+0+0+0+0+0+0+0=0
\end{aligned}
$$




$$
\begin{aligned}
& y=\left[\begin{array}{ccc}
1 & \llbracket-3 \rrbracket & 0 \\
2 & 4 & 0 \\
0 & 0 & 0
\end{array}\right] \times\left[\begin{array}{ccc}
2 & \llbracket 0 \rrbracket & 0 \\
0 & 0 & 0 \\
1 & -5 & 0
\end{array}\right]=2+0+0+0+0+0+0+0+0=2 \\
& y=\left[\begin{array}{ccc}
1 & \llbracket-3 \rrbracket & 0 \\
2 & 4 & 0 \\
0 & 0 & 0
\end{array}\right] \times\left[\begin{array}{ccc}
1 & -5 & 0 \\
2 & \llbracket 0 \rrbracket & 0 \\
0 & 0 & 0
\end{array}\right]=1+15+0+4+0+0+0+0+0=20 \\
& y=\left[\begin{array}{ccc}
1 & \llbracket-3 \rrbracket & 0 \\
2 & 4 & 0 \\
0 & 0 & 0
\end{array}\right] \times\left[\begin{array}{ccc}
0 & 1 & -5 \\
0 & 2 & \llbracket 0 \rrbracket \\
0 & 0 & 0
\end{array}\right]=0-3+0+0+8+0+0+0+0=5 \\
& y=\left[\begin{array}{ccc}
1 & \llbracket-3 \rrbracket & 0 \\
2 & 4 & 0 \\
0 & 0 & 0
\end{array}\right] \times\left[\begin{array}{ccc}
0 & 0 & 0 \\
0 & 1 & -5 \\
0 & 2 & \llbracket 0 \rrbracket
\end{array}\right]=0+0+0+0+4+0+0+0+0=4 \\
& y=\left[\begin{array}{ccc}
1 & \llbracket-3 \rrbracket & 0 \\
2 & 4 & 0 \\
0 & 0 & 0
\end{array}\right] \times\left[\begin{array}{ccc}
0 & 2 & \llbracket 0 \rrbracket \\
0 & 0 & 0 \\
0 & 1 & -5
\end{array}\right]=0-6+0+0+0+0+0+0+0=-6
\end{aligned}
$$

Thus, according to start point location of $h$ matrix (i.e. [[0]]) $y$ matrix is

$$
y=\left[\begin{array}{ccc}
0 & \llbracket 2 \rrbracket & -6 \\
-5 & 20 & 5 \\
-10 & -18 & 4
\end{array}\right]
$$

While if $y$ is a matrix and it is equal to circular convolution of $x$ with $h$ as follows

$$
\begin{aligned}
y(n 1, n 2) & =\sum_{k 1}^{N 1-1} \sum_{k 2}^{N 2-1} x(k 1, k 2) h[(n 1-k 1, n 2-k 2) \bmod (N 1, N 2)] \\
\text { or } \quad y(n 1, n 2) & =x(n 1, n 2) * h[(n 1, n 2) \bmod (N 1, N 2)]
\end{aligned}
$$

It clears that $N 1$ and $N 2$ are equal to two for circular correlation, in matrix $y$. according to the fast convolution algorithm the solution as follows:

$$
\begin{aligned}
& x=\left[\begin{array}{cc}
1 & \llbracket-3 \rrbracket \\
2 & 4
\end{array}\right], h=\left[\begin{array}{ll}
{[0 \rrbracket} & 2 \\
-5 & 1
\end{array}\right] \text { and } h \text { reverse is, } h=\left[\begin{array}{cc}
1 & -5 \\
2 & \llbracket 0 \rrbracket
\end{array}\right], \text { therefore } \\
& y=\left[\begin{array}{cc}
1 & \llbracket-3 \rrbracket \\
2 & 4
\end{array}\right] \times\left[\begin{array}{cc}
1 & -5 \\
2 & \llbracket 0 \rrbracket
\end{array}\right]=1+15+4+0=20 \\
& y=\left[\begin{array}{cc}
1 & \llbracket-3 \rrbracket \\
2 & 4
\end{array}\right] \times\left[\begin{array}{cc}
-5 & 1 \\
\llbracket 0 \rrbracket & 2
\end{array}\right]=-5-3+0+8=0 \\
& y=\left[\begin{array}{cc}
1 & \llbracket-3 \rrbracket \\
2 & 4
\end{array}\right] \times\left[\begin{array}{ll}
{[0 \rrbracket} & 2 \\
-5 & 1
\end{array}\right]=0-6-10+4=-12 \\
& y=\left[\begin{array}{cc}
1 & \llbracket-3 \rrbracket \\
2 & 4
\end{array}\right] \times\left[\begin{array}{cc}
2 & \llbracket 0 \rrbracket \\
1 & -5
\end{array}\right]=2+0+2-20=-16 \\
& y=\left[\begin{array}{cc}
-12 & \llbracket-16 \rrbracket \\
0 & 20
\end{array}\right]
\end{aligned}
$$

The arrangement numbers in $y$ matrix is according to location of start point of $h$ matrix (I0I). Note, by folding the answer of linear convolution according to length of circular convolution it produces the answer of circular convolution (see Figure 1): 


\section{Fast Correlation 2-D:}

Assume the two matrices below:

$$
x=\left[\begin{array}{cc}
1 & \llbracket-3 \rrbracket \\
2 & 4
\end{array}\right] \text { and } h=\left[\begin{array}{cc}
\llbracket 0 \rrbracket & 2 \\
-5 & 1
\end{array}\right]
$$

In addition, $\mathrm{y}$ is a matrix and it is equal to linear correlation of $x$ with $h$ as follows

$$
y(n 1, n 2)=\Sigma_{k 1} \Sigma_{k 2} x(k 1, k 2) h(n 1+k 1, n 2+k 2)
$$

It clears that number of rows and columns are equal to three, in matrix $y$. So according to the fast correlation algorithm the solution is as follows:

$$
\begin{aligned}
& x=\left[\begin{array}{ccc}
1 & \llbracket-3 \rrbracket & 0 \\
2 & 4 & 0 \\
0 & 0 & 0
\end{array}\right] \text { and } h=\left[\begin{array}{ccc}
0 & \llbracket 0 \rrbracket & 2 \\
0 & -5 & 1 \\
0 & 0 & 0
\end{array}\right] \text {, so } \\
& y=\left[\begin{array}{ccc}
1 & \llbracket-3 \rrbracket & 0 \\
2 & 4 & 0 \\
0 & 0 & 0
\end{array}\right] \times\left[\begin{array}{ccc}
0 & \llbracket 0 \rrbracket & 2 \\
0 & -5 & 1 \\
0 & 0 & 0
\end{array}\right]=0+0+0+0-20+0+0+0+0=-20 \\
& y=\left[\begin{array}{ccc}
1 & \llbracket-3 \rrbracket & 0 \\
2 & 4 & 0 \\
0 & 0 & 0
\end{array}\right] \times\left[\begin{array}{ccc}
0 & 0 & 0 \\
0 & \llbracket 0 \rrbracket & 2 \\
0 & -5 & 1
\end{array}\right]=0+0+0+0+0+0+0+0+0=0 \\
& y=\left[\begin{array}{ccc}
1 & \llbracket-3 \rrbracket & 0 \\
2 & 4 & 0 \\
0 & 0 & 0
\end{array}\right] \times\left[\begin{array}{ccc}
0 & -5 & 1 \\
0 & 0 & 0 \\
0 & \llbracket 0 \rrbracket & 2
\end{array}\right]=0+15+0+0+0+0+0+0+0=15 \\
& y=\left[\begin{array}{ccc}
1 & \llbracket-3 \rrbracket & 0 \\
2 & 4 & 0 \\
0 & 0 & 0
\end{array}\right] \times\left[\begin{array}{ccc}
-5 & 1 & 0 \\
0 & 0 & 0 \\
\llbracket 0 \rrbracket & 2 & 0
\end{array}\right]=-5-3+0+0+0+0+0+0+0=-8 \\
& y=\left[\begin{array}{ccc}
1 & \llbracket-3 \rrbracket & 0 \\
2 & 4 & 0 \\
0 & 0 & 0
\end{array}\right] \times\left[\begin{array}{ccc}
0 & 0 & 0 \\
{[0 \rrbracket} & 2 & 0 \\
-5 & 1 & 0
\end{array}\right]=0+0+0+0+8+0+0+0+0=8 \\
& y=\left[\begin{array}{ccc}
1 & \llbracket-3 \rrbracket & 0 \\
2 & 4 & 0 \\
0 & 0 & 0
\end{array}\right] \times\left[\begin{array}{ccc}
{[0 \rrbracket} & 2 & 0 \\
-5 & 1 & 0 \\
0 & 0 & 0
\end{array}\right]=0-6+0-10+4+0+0+0+0=-12 \\
& y=\left[\begin{array}{ccc}
1 & \llbracket-3 \rrbracket & 0 \\
2 & 4 & 0 \\
0 & 0 & 0
\end{array}\right] \times\left[\begin{array}{ccc}
2 & 0 & \llbracket 0 \rrbracket \\
1 & 0 & -5 \\
0 & 0 & 0
\end{array}\right]=2+0+0+2+0+0+0+0+0=4 \\
& y=\left[\begin{array}{ccc}
1 & \llbracket-3 \rrbracket & 0 \\
2 & 4 & 0 \\
0 & 0 & 0
\end{array}\right] \times\left[\begin{array}{ccc}
0 & 0 & 0 \\
2 & 0 & \llbracket 0 \rrbracket \\
1 & 0 & -5
\end{array}\right]=0+0+0+4+0+0+0+0+0=4 \\
& y=\left[\begin{array}{ccc}
1 & \llbracket-3 \rrbracket & 0 \\
2 & 4 & 0 \\
0 & 0 & 0
\end{array}\right] \times\left[\begin{array}{ccc}
1 & 0 & -5 \\
0 & 0 & 0 \\
2 & 0 & \llbracket 0 \rrbracket
\end{array}\right]=1+0+0+0+0+0+0+0+0=1
\end{aligned}
$$

Thus, according to location of start point of $h$ matrix ([I0]). $y$ matrix is 
$y=\left[\begin{array}{ccc}-12 & \llbracket-20 \rrbracket & 4 \\ 8 & 0 & 4 \\ -8 & 15 & 1\end{array}\right]$

While if $y$ is a matrix and it is equal to circular correlation of $x$ with $h$ as follows

$$
y(n 1, n 2)=\Sigma_{k 1}^{N 1-1} \sum_{k 2}^{N 2-1} x(k 1, k 2) h[(n 1+k 1, n 2+k 2) \bmod (N 1, N 2)]
$$

It clears that $N 1$ and $N 2$ are equal to two for circular correlation, in matrix $y$. According to the fast correlation algorithm the solution as follows:

$$
\begin{aligned}
& x=\left[\begin{array}{cc}
1 & \llbracket-3 \rrbracket \\
2 & 4
\end{array}\right] \text { and } h=\left[\begin{array}{cc}
\llbracket 0 \rrbracket & 2 \\
-5 & 1
\end{array}\right] \text {, so } \\
& y=\left[\begin{array}{cc}
1 & \llbracket-3 \rrbracket \\
2 & 4
\end{array}\right] \times\left[\begin{array}{cc}
{[0 \rrbracket} & 2 \\
-5 & 1
\end{array}\right]=0-6-10+4=-12 \\
& y=\left[\begin{array}{cc}
1 & \llbracket-3 \rrbracket \\
2 & 4
\end{array}\right] \times\left[\begin{array}{cc}
2 & \llbracket 0 \rrbracket \\
1 & -5
\end{array}\right]=2+0+2-20=-16 \\
& y=\left[\begin{array}{cc}
1 & \llbracket-3 \rrbracket \\
2 & 4
\end{array}\right] \times\left[\begin{array}{cc}
1 & -5 \\
2 & \llbracket 0 \rrbracket
\end{array}\right]=1+15+4+0=20 \\
& y=\left[\begin{array}{cc}
1 & \llbracket-3 \rrbracket \\
2 & 4
\end{array}\right] \times\left[\begin{array}{cc}
-5 & 1 \\
\llbracket 0 \rrbracket & 2
\end{array}\right]=-5-3+0+8=0 \\
& y=\left[\begin{array}{cc}
-12 & \llbracket-16 \rrbracket \\
0 & 20
\end{array}\right]
\end{aligned}
$$

The arrangement numbers in $y$ matrix is according to location of start point of $h$ matrix (I0I). Note, by folding the answer of linear correlation according to length of circular correlation it produces the answer of circular correlation (see Figure 2).

\section{Fast Convolution 3-D:}

Assume the two matrices below:

$x=\begin{array}{cc}1 & 3 \\
\llbracket 2 \rrbracket & 1\end{array} \quad$ and $h=$\begin{tabular}{cc|}
-1 & 4 \\
$\llbracket 5 \rrbracket$ & 7 \\
\hline
\end{tabular}

In addition, $\mathrm{y}$ is a matrix and it is equal to linear convolution of $x$ with $h$ as follows

$$
\begin{aligned}
y(n 1, n 2, n 3) & =\sum_{k 1} \sum_{k 2} \sum_{k 3} x(k 1, k 2, k 3) h(n 1-k 1, n 2-k 2, n 3-k 3) \\
\text { or } \quad y(n 1, n 2, n 3) & =x(n 1, n 2, n 3) * * n(n 1, n 2, n 3)
\end{aligned}
$$

Where the symbol *** represents the 3-D discrete convolution. It clears that number of rows; columns and depths are equal to three, in matrix $y$. So according to the fast convolution algorithm the solution as follows:

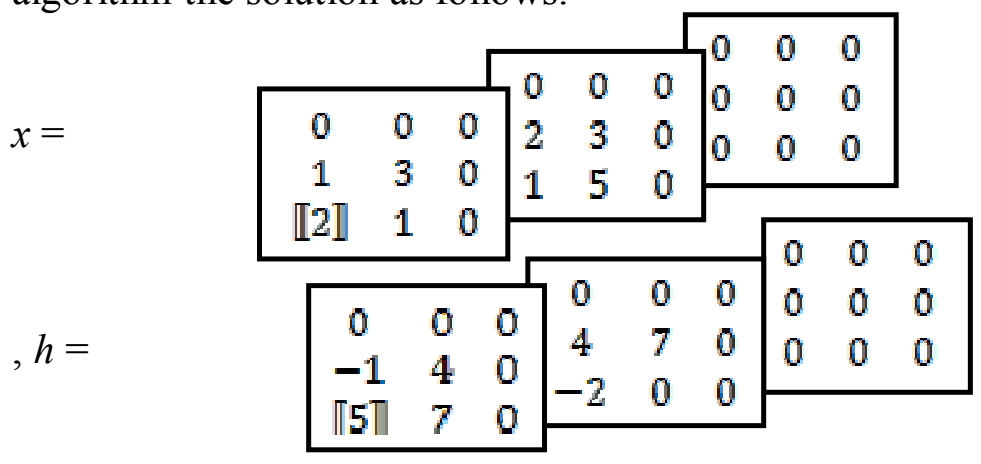




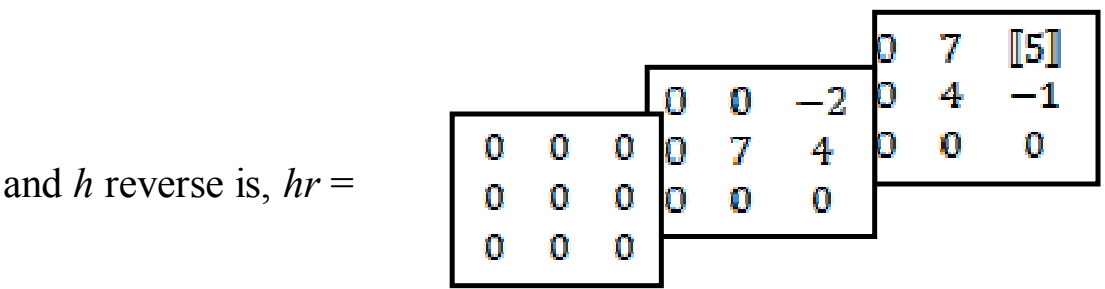

When multiply $x$ with $h r$, the sub-matrix ( $3^{\text {rd }}$ square) will be produced (i.e. $n 1$ and $n 2=1,2$ and 3 , while $n 3=3$ only) according to location of starting point [[5]].

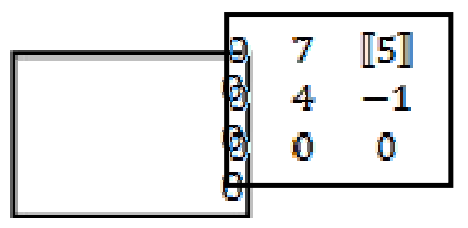

$y=x * h r=x *$

$y=0+0+0+0+21+0+0+0+0=21$

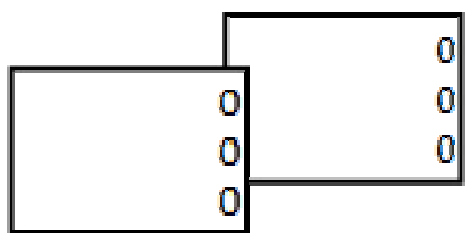

$y=x * h r=x *$

$y=0+0+0+0+0+0+0+35+0=35$

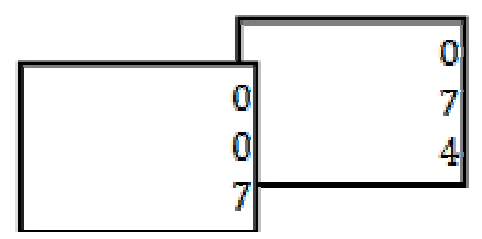

$y=x * h r=x *$

$y=0+0+0+0-6+0+7+20+0=21$

Therefore, continue to the end of sub-matrix when $n 3=3$. Hence, each result has a location according to location of $[[5]]$ and results are $(8,26,21,21,0,-10,0,35$, and -2$)$. Now rotate the matrix $h r$ one-step clockwise direction (or counter clockwise with staying on same direction to end process) according to the following figure: 
For each case, there is produced one-way direction as above when $n 3=3$, then the sub-matrix $\left(1^{\text {st }}\right.$ square $)$ will compute as shown below:

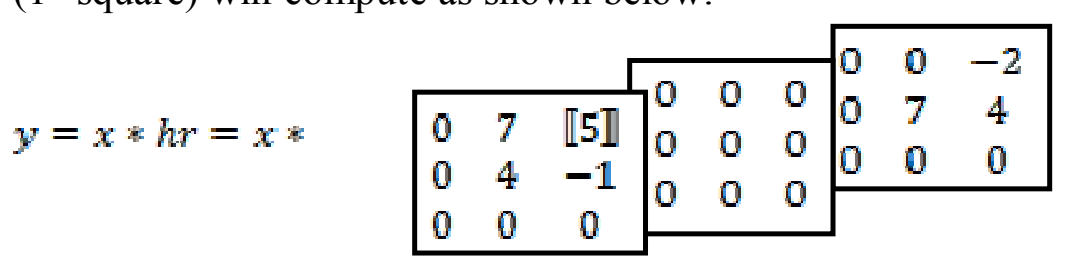$$
y=0+0+0+0+12+0+0+0+0=12
$$$$
y=x * h x=x * \begin{array}{ccc|ccc|ccc|}
0 & 0 & 0 & -2 & 0 \\
7 & \llbracket 5 \rrbracket & 0 & 0 & 0 \\
4 & -1 & 0 & 0 & 0 & 0 & 0 \\
0 & 0 & 0 & 0 & 0 & 0 & & &
\end{array}
$$

$y=0+0+0+4-3+0+0+0+0=1$

Therefore, continue to the end of sub-matrix when $n 3=1$. Hence, each result has a location according to location of [[5]] and results are $(-1,25,29,1,10,19,3,7$, and 12). Now rotate the matrix $h r$ other one-step clockwise direction to produce last sub-matrix $(n 3=2)$.

$$
y=x * h r=x * \begin{array}{cccccc|ccc|}
n & 0 & -2 & 0 & 7 & \text { I5】 } & 0 & 0 \\
0 & 0 & 0 \\
0 & 7 & 4 & 0 & 4 & -1 & 0 & 0 & 0 \\
0 & 0 & 0 & 0 & 0 & & &
\end{array}
$$

$y=0+0+0+0+21+0+0+0+0+0+0+0+0+12+0+0+0+0=33$

$$
y=x * h r=x * \begin{array}{cccc|ccc|ccc|}
n & 0 & 0 & 0 & 0 & 0 & 0 & 0 \\
0 & 0 & 0 \\
0 & 0 & -2 & 0 & 7 & \llbracket 5 \rrbracket & 0 & 0 & 0 \\
0 & 7 & 4 & 0 & 4 & -1 & & & &
\end{array}
$$

$$
y=0+0+0+0+21+0+0+20+0+0+0+0+0+0+0+0+7+0=48
$$

Therefore, continue to the end of sub-matrix when $n 3=2$. Hence, each result has a location according to location of $[[5]]$ and results are $(2,24,33,15,40,35,30,1$, and 48). Finally, the result is

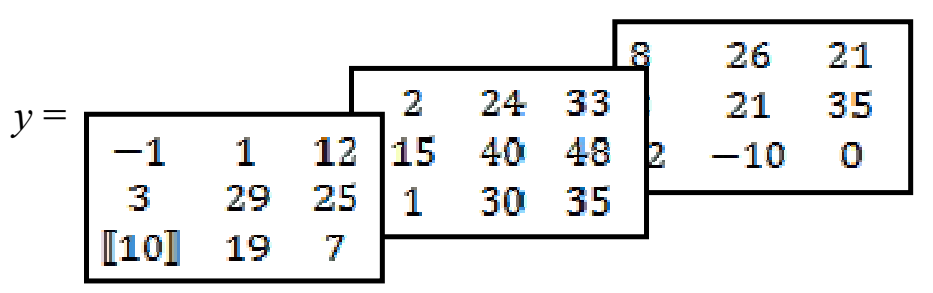

While if $y$ is a matrix and it is equal to circular convolution of $x$ with $h$ as follows 
$y(n 1, n 2, n 3)=$

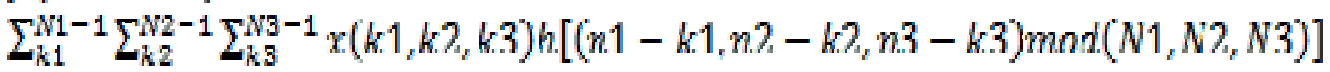

or $\quad y(n 1, n 2, n 3)=x(n 1, n 2, n 3) \cdots=h[(n 1, n 2, n 3) \bmod (N 1, N 2, N 3)]$

It clears that $N 1, N 2$, and $N 3$ are equal to two for circular correlation, in matrix $y$. According to the fast convolution algorithm the solution as follows:

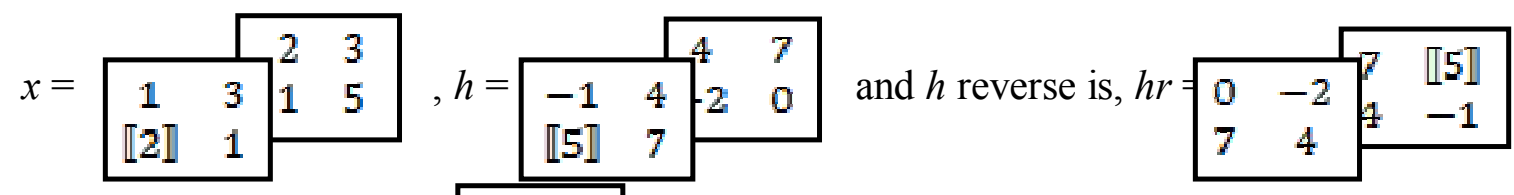

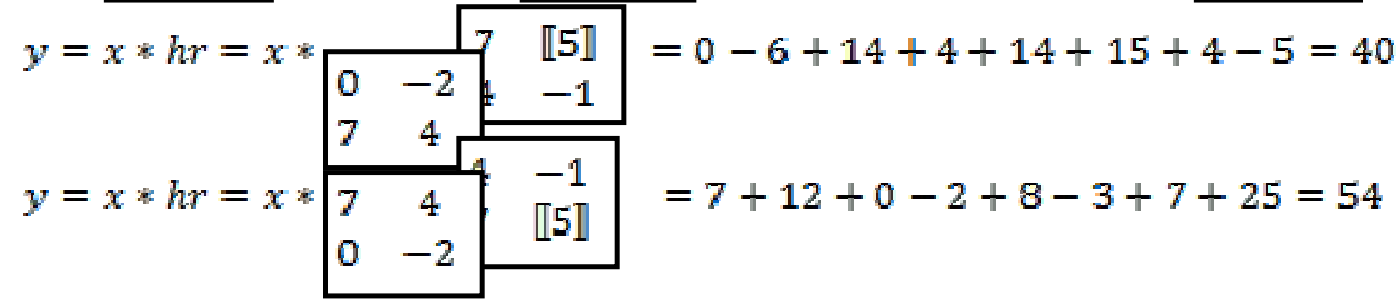

herefore, continue for all location. Hence, each result has a location according to location of [[5]] and results are $(54,63,63,40,55,50,71$, and 36). Finally, the result is

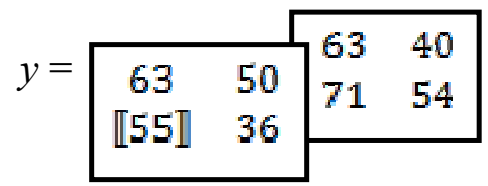

Note, by folding the answer of linear convolution according to length of circular convolution it produces the answer of circular convolution as the figure(5).

\section{FAST CORRELATION 3-D:}

Assume the two matrices below:

$x=\begin{array}{cc}1 & 3 \\ \llbracket 2 \rrbracket & \mathbb{1}\end{array} \quad$ and $h=\begin{array}{|cc|}-1 & 4 \\ \llbracket 5 \rrbracket & 7\end{array}$

In addition, $\mathrm{y}$ is a matrix and it is equal to linear correlation of $x$ with $h$ as follows

$$
y(n 1, n 2, n 3)=\sum_{k 1} \sum_{k 2} \sum_{k 3} x(k 1, k 2, k 3) h(n 1+k 1, n 2+k 2, n 3+k 3)
$$

It clears that number of rows; columns and depths are equal to three, in matrix $y$. So according to the fast correlation algorithm the solution as follows:
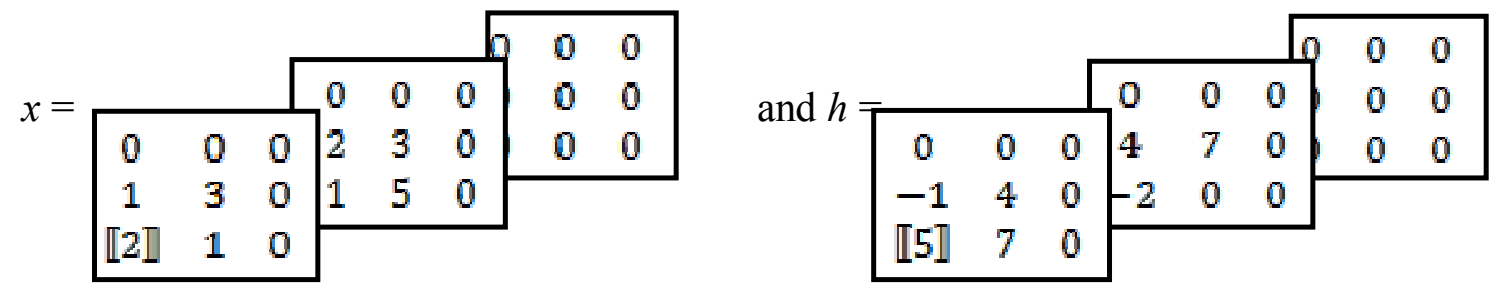
When multiply $x$ with $h$, the sub-matrix ( $1^{\text {st }}$ square) will be produced (i.e. $n 1$ and $n 2=1,2$ and 3 , while $n 3=1$ only) according to location of starting point [[5]].

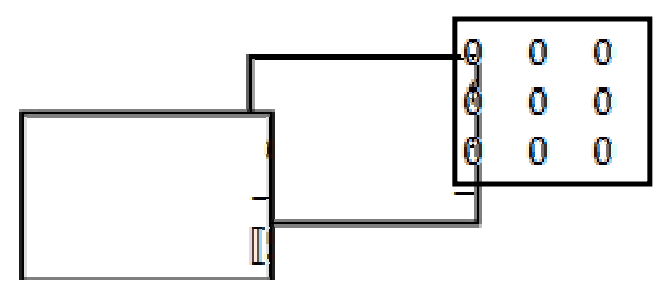

$y=x * h=x *$

$y=0+0+0-1+12+0+10+7+0+0+0+0+8+21+0-2+0+0=55$

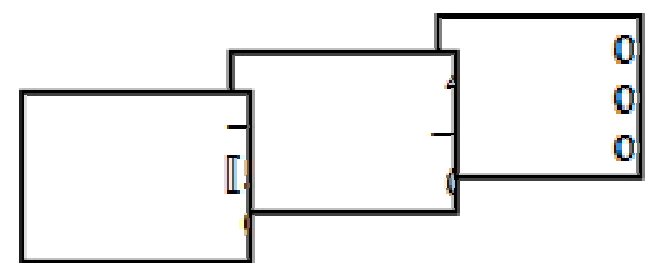

$y=x * h=x *$

$y=0+0+0+5+21+0+0+0+0+0+0+0-4+0+0+0+0+0=22$
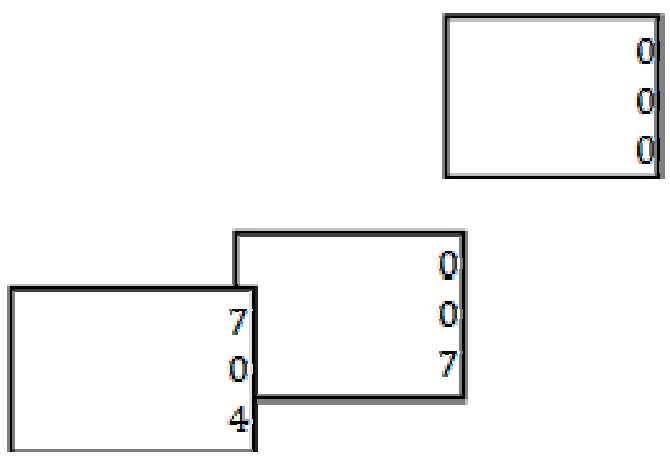

$y=x * h=x *$

$y=0+0+0+0+0+0+8+0+0+0+0+0+0+0+0+7+0+0=15$

Therefore, continue to the end of sub-matrix when $n 3=1$. Hence, each result has a location according to location of [[5]] and results are $(55,22,41,19,9,4,32,15$, and 7). Now rotate the matrix $h$ one-step clockwise direction (or counter clockwise with staying on same direction to end process) as Figure 3.

For each case, there is produced one-way direction as above when $n 3=1$, then the sub-matrix $\left(2^{\text {nd }}\right.$ square) will compute as shown below:

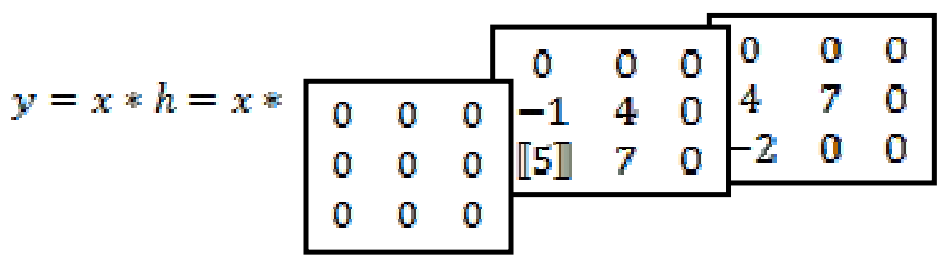


$y=0+0+0-2+12+0+5+35+0=50$

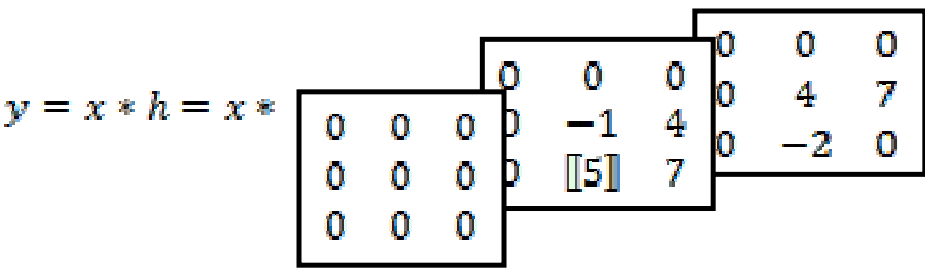

$y=0+0+0+0-3+0+0+25+0=22$

Therefore, continue to the end of sub-matrix when $n 3=2$. Hence, each result has a location according to location of [[5]] and results are $(-5,22,31,15,50,19,4,15$, and 14). Now rotate the matrix $h$ other one-step clockwise direction to produce last sub-matrix $(n 3=3)$.

$y=x * h=x *$\begin{tabular}{cccccc|ccc|}
$n$ & & & 0 & 0 & 0 \\
0 & 0 & 0 & 0 & 0 & -1 & 4 & 0 \\
4 & 7 & 0 & 0 & 0 & 0 & 0 \\
-2 & 0 & 0 & 151 & 7 & 0 \\
\hline
\end{tabular}

$y=0+0+0+4+21+0-4+0+0=21$

$y=x * h=x *$\begin{tabular}{ccc|ccc|ccc|}
$n$ & & & 0 & 0 & -1 & 4 & 0 \\
4 & 7 & 0 & 0 & 0 & 0 & {$[5]$} & 7 & 0 \\
-2 & 0 & 0 & 0 & 0 & 0 & 0 & 0 & 0 \\
0 & 0 & 0 & & & &
\end{tabular}

$y=0+0+0-2+0+0+0+0+0=-2$

Therefore, continue to the end of sub-matrix when $n 3=3$. Hence, each result has a location according to location of [[5]] and results are $(15,4,14,0,-6,-2,10,7$, and 21). Finally, the result is

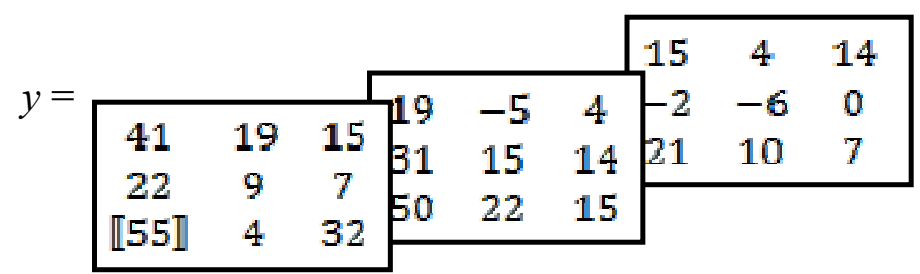

While if $y$ is a matrix and it is equal to circular correlation of $x$ with $h$ as follows

$y(n 1, n 2, n 3)=$

$\sum_{k 1}^{N 1-1} \sum_{k 2}^{N 2-1} \sum_{k 3}^{N 3-1} x(k 1, k 2, k 3) h[(n 1+k 1, n 2+k 2, n 3+k 3) \bmod (N 1, N 2, N 3)]$

It clears that $N 1, N 2$, and $N 3$ are equal to two for circular correlation, in matrix $y$. According to the fast correlation algorithm the solution as follows:

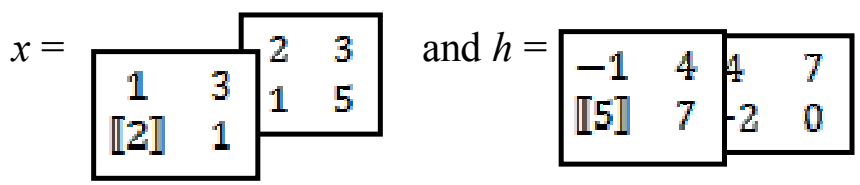




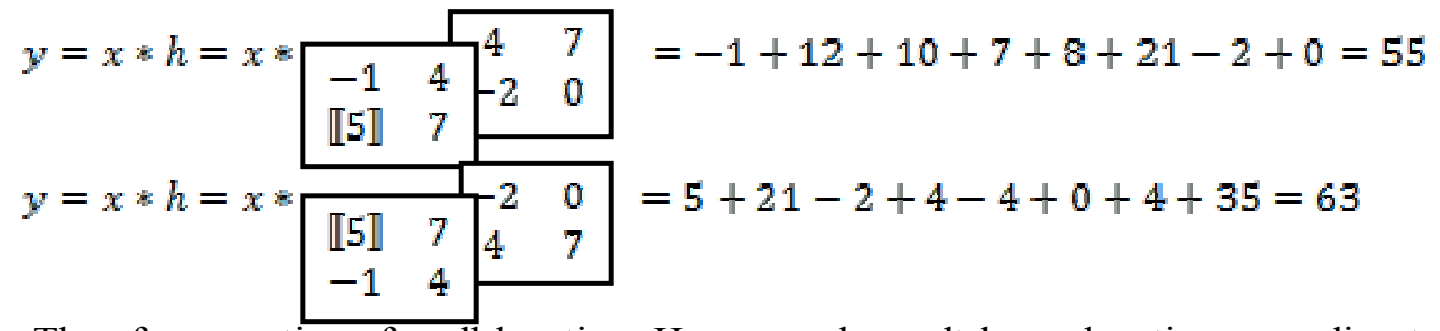

Therefore, continue for all location. Hence, each result has a location according to location of $[[5]]$ and results are $(54,63,63,40,55,50,71$, and 36). Finally, the result is

$y=$\begin{tabular}{cc|cc|}
63 & 50 & 63 & 40 \\
$\llbracket 55 \rrbracket$ & 36 & 71 & 54 \\
\hline
\end{tabular}

Note, by folding the answer of linear correlation according to length of circular correlation it produces the answer of circular convolution as Figure 5.

\section{CONCLUSIONS}

The proposed algorithm is faster than the traditional algorithms and it is easy by hand and computer, which is clearing in the different examples. In addition, it is easy to locate the start sample and locate the other samples from the start sample of one of the two matrices according to section two, because the traditional algorithms does not computing easily for signals of greater than or equal to 2-D. Finally, the proposed algorithm can be applying on the signals of any dimension 1-D, 2-D, 3-D...etc and these signals not necessary to be a square matrix.

\section{REFERENCES}

1. Smith S.W., (2003), "Digital Signal Processing A Practical Guide for Engineers and Scientists," Newnes, USA.

2. Tan L., (2008), "Digital Signal Processing Fundamentals and Applications," Elsevier, USA.

3. Madisetti V.K., (2010), "Digital Signal Processing Fundamentals," Taylor and Francis Group, USA.

4. Sabin W.E., (2008), "Discrete-Signal Analysis and Design," John Wiley \& Sons, USA.

5. Proakis J.G., \& Manolakis D.G., (1996), "Digital Signal Processing Principles, Algorithms, and Applications," Prentice Hall International, USA.

6. Parker M., (2010), "Digital Signal Processing Everything you need to know to get started," Elsevier, USA. 
7. Mahmoud W.A., (2001), "A New Multi- Dimensional Convolution Algorithm for Digital Signals," Computer, Communication, Control and System Engineering Magazine, Vol.2, No.2, PP. 72-78.

8. Kumar B.P., (2005), "Digital Signal Processing Laboratory," Taylor and Francis Group, USA.

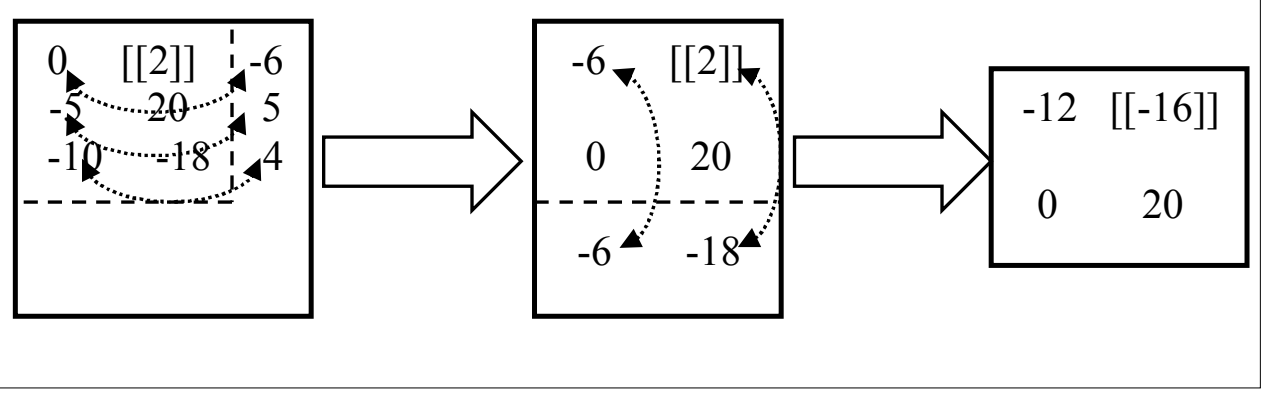

Fig.( 1): Converting the linear convolution answer to the circular convolution answer by folding for 2-D signals.

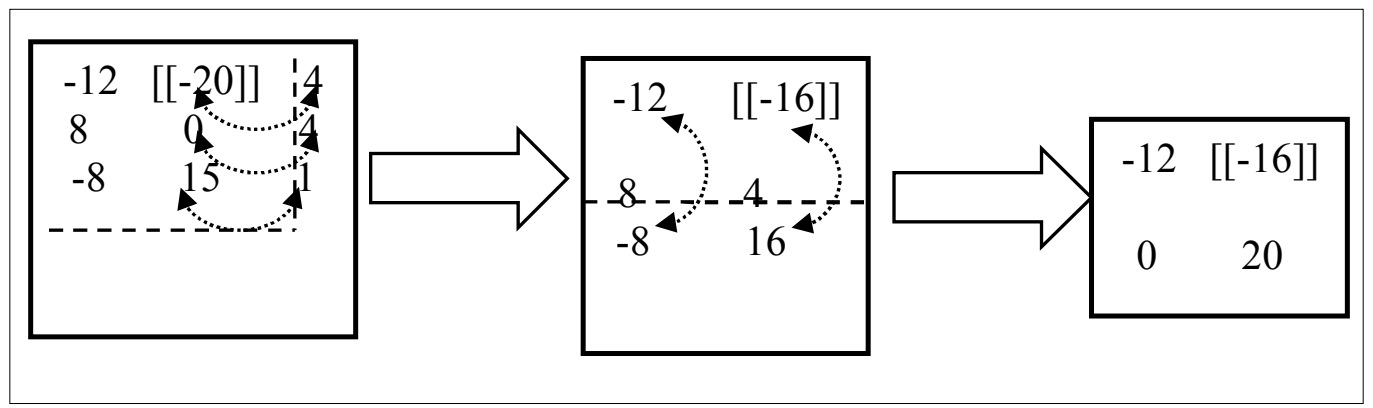

Fig.(2): Converting the linear correlation answer to the circular correlation answer by folding for 2-D signals. 


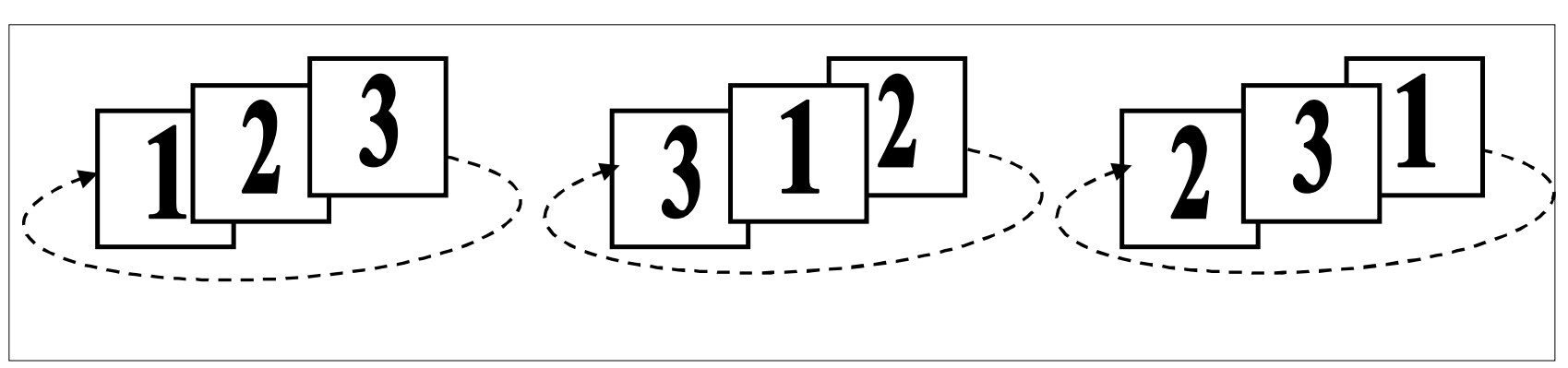

Fig.(3): Rotation direction of 3-D signals when convolution calculation.

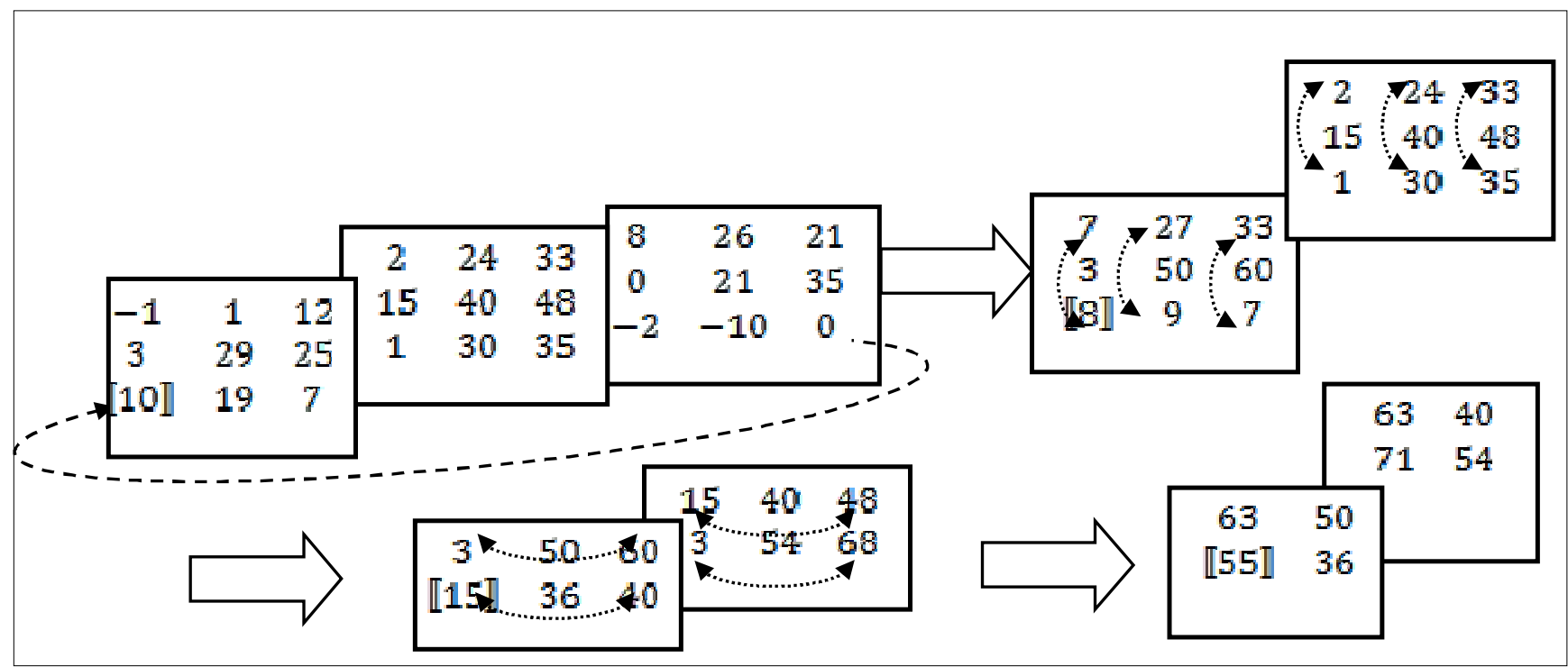

Fig.(4): Converting the linear convolution answer to the circular convolution answer by folding for 3-D signals.

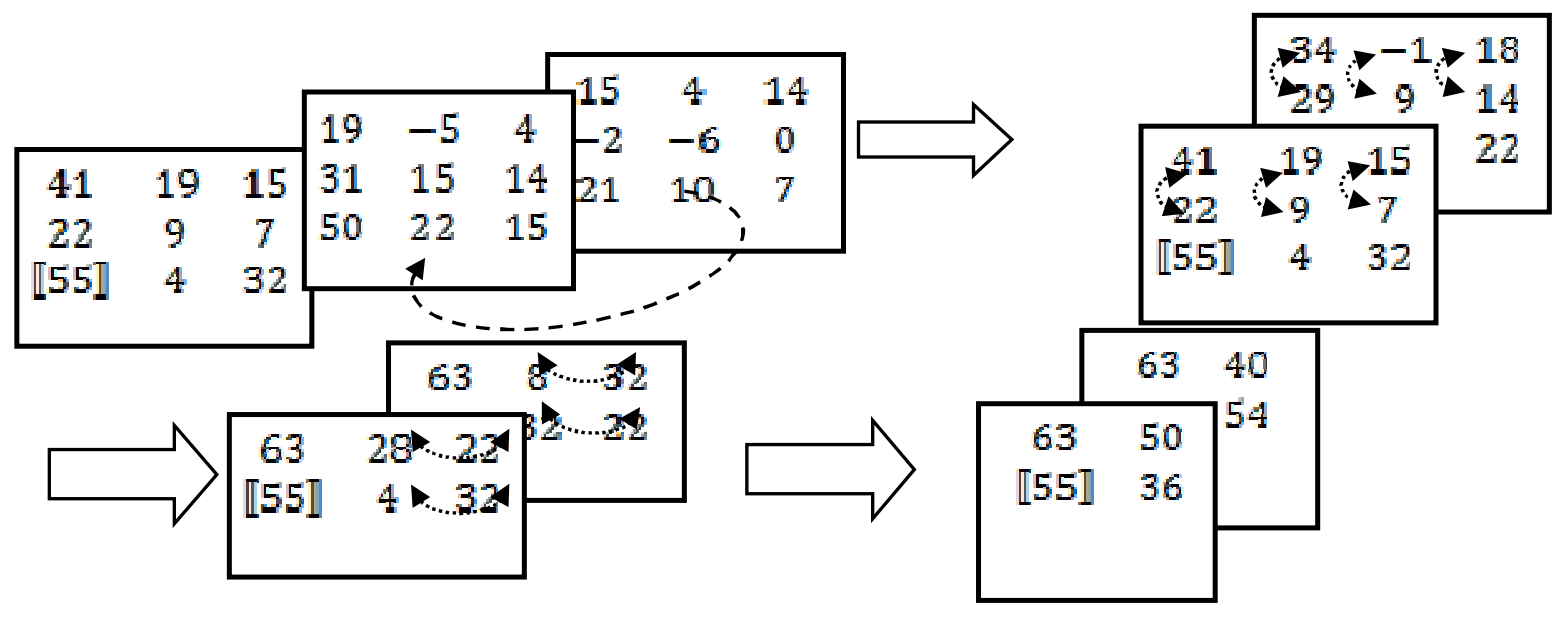

Fig.(5): Converting the linear correlation answer to the circular correlation answer by folding for 3-D signals. 
الخوارزمية السريعة لحساب التكامل الالتفافي والتعاقبي للاشارات المتعددة الابعاد

\author{
عماد حمود سلمان \\ المدرس المساعد \\ كلية الهندسة، جامعة ديالى
}

الخلاصــة

العالم التقني يتغير بسرعة كبيرة جدا. في العقود الثناثة الاخيرة، الحواسيب الثخصية ازدادت بمقدار القوة الف.

بكل الحسابات، العدد سيزداد في العقد القادم بالقوة الف مرة اخرى. هذه القوة القابلة للزيادة طورت طرق العلوم والهندسة، ولايوجد هناك افضل مثالا من معالج الاشارة الرقمية.

هذا العمل المقترح هو خوارزمية سريعة لحساب بعض العمليات الرياضية المهمة في معالج الاشارة الرقمية، واللتان هما التكامل الالتفافي والتكامل التعاقبي (كلاهما خطي ودوراني). هذه العمليات تدخل في معظم التطبيقات اليومية

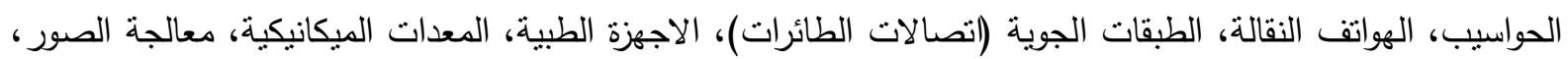
المؤتمرات المتلفزة، واخرى. لذلك، للحصول على نظام ذو زمن حقيقي (هذا يعني لنقليل زمن التأخير لأي منظومة). 